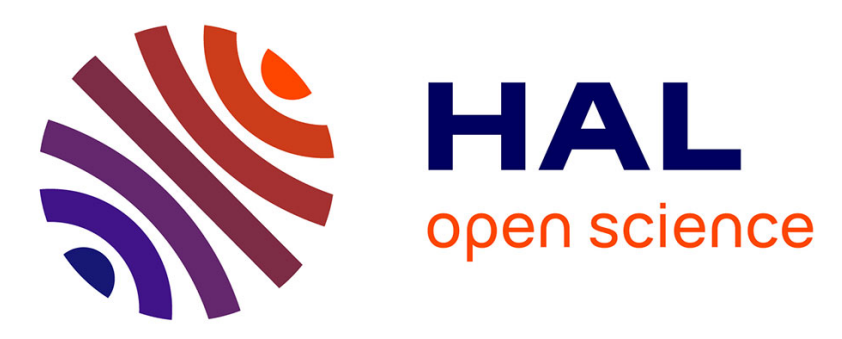

\title{
Numerical and experimental investigation of the cavitating flow within Venturi tube
}

Jiří Kozák, Pavel Rudolf, Martin Hudec, David Štěfan, Matěj Forman

\section{To cite this version:}

Jiří Kozák, Pavel Rudolf, Martin Hudec, David Štěfan, Matěj Forman. Numerical and experimental investigation of the cavitating flow within Venturi tube. ISROMAC 2017 International Symposium on Transport Phenomena and Dynamics of Rotating Machinery, Dec 2017, Maui, United States. hal02369311

\section{HAL Id: hal-02369311 \\ https://hal.science/hal-02369311}

Submitted on 18 Nov 2019

HAL is a multi-disciplinary open access archive for the deposit and dissemination of scientific research documents, whether they are published or not. The documents may come from teaching and research institutions in France or abroad, or from public or private research centers.
L'archive ouverte pluridisciplinaire HAL, est destinée au dépôt et à la diffusion de documents scientifiques de niveau recherche, publiés ou non, émanant des établissements d'enseignement et de recherche français ou étrangers, des laboratoires publics ou privés.

\section{(c)(1)}

Distributed under a Creative Commons Attribution| 4.0 International License 


\title{
Numerical and experimental investigation of the cavitating flow within Venturi tube
}

\author{
Jiří Kozák $^{1 *}$, Pavel Rudolf ${ }^{1}$, Martin Hudec $^{1}$, David Štěfan ${ }^{1}$, Matěj Forman ${ }^{2}$
}

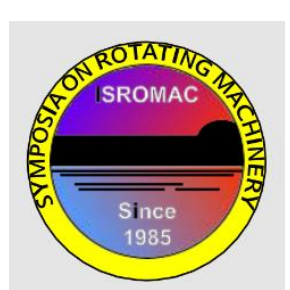

ISROMAC 2017

International

Symposium on Transport

Phenomena and

Dynamics of

Rotating Machinery

Hawaii, Maui

December 16-21 2017

\begin{abstract}

\author{
Keywords \\ Cavitaton — Swirl — Venturi tube-OpenFoam \\ ${ }^{1}$ Victor Kaplan dpt. Of Fluid Eng., Brno University of Technology, Czech Republic \\ ${ }^{2}$ ESI Group, Czech Republic \\ *Corresponding author: jiri.kozak1@gmail.com
}

Hydrodynamic cavitation represents complex physical phenomenon undesirably affecting operation as well as lifespan of many hydraulic machines from small valves to the large hydro power plants. On the other hand, the same phenomenon and its concomitants such as pressure pulsations can be exploited in many positive ways. One of them which seems to be very promising and perspective is the cavitation utilization for reduction of the microorganisms such as cyanobacteria within large bulks of water. Mutual effect of the swirl induced by the upstream mounted generator and flow constriction in convergingdiverging nozzle has been experimentally investigated. The main scope of this paper is numerical investigation complementing the experimental results. The multiphase simulations were carried out using the OpenFoam 1606+ and its interPhaseChangeFoam solver. The present study focuses on CFD results of the cavitating velocity field within the nozzle and analysis of the loss coefficient within the nozzle.

\section{INTRODUCTION}

The investigation of the cavitation is one of the key research directions of hydraulic engineering and multiphase fluid mechanics in general. In case of the pressure drop below the value of saturated water vapor, cavities filled mainly by the vapor occurred within the liquid. One of the most important type of cavitation in the field of hydraulic engineering is represented by so-called hydrodynamic cavitation, where the pressure drop is induced by the local increase of the flow velocity. [1]

Vapor cavities within the flow induce pressure pulsations, vibrations and noise. Above that, the surfaces of hydraulic machines and pipelines exposed to the collapsing vapor cavities are threatened by so-called cavitation erosion due to the enormous local variations of pressure and temperature. These reasons led to exhaustive research of this phenomenon from the beginning of the last century to the present days. Since the abovementioned concomitants of cavitation are usually undesirable, the research of this phenomenon is focused mainly on the cavitation suppression.

Typical example could be investigation of the off-design operation of the hydraulic turbines. [2] This issue has gained importance mainly with the expansion of renewable sources, where hydro power plants play important role in the electrical grid regulation. On the other hand, the off-design operation of certain hydraulic turbines types leads to the inception of the vortex structures downstream the turbine runner due to the presence of the significant residual swirl within the flow. This problem is particularly severe in case of Francis turbines, due to the fixed angle of the runner blades and frequent utilization of this type of hydraulic turbine.
Cavitating vortex rope is mostly present due to the pressure drop in the core of the swirl. This reasons evoked extensive investigation of this problematics. Since the investigation on the full-size turbines represents considerable finance burden and even the utilization of scaled down turbine models can be unbearably expensive, the exploitation of so-called swirl generators is common practice. [3], [13]

The swirl generator designed for this particular application, specifically for the investigation of the swirl decay and dissipation within the diffuser, has been exploited for the investigation of the cavitation induced by the swirl within the Venturi tube. A Venturi tube is a converging-diverging nozzle with short straight throat in between both sections. Investigation is motivated mainly by the idea to apply hydrodynamic cavitation for cyanobacteria elimination [4] or generally for disinfection of large bulks of water. This application of the cavitation seems to be effective and above that, thanks to its physical nature (e.g. absence of chemical agents), environmentally-friendly. This is particularly important in case of water reservoirs which are used for the recreation or as a drinking water sources.

While the principle of the cavitation reduction of the cyanobacteria within the water seems to be an effective approach, the complete mechanism of the disruption of this organism has to be analyzed. For this purpose, cavitation and its dynamics within the Venturi tube have been investigated and results were discussed in several contributions. [5][6][7]

Introduction of swirl using the swirl generator represents the further step of this investigation. The cavitation patterns downstream the throat of the nozzle have been highly modified by the presence of the swirl. The additional pressure drop causes step up of the cavitation amount. Above that the 
redistribution of the flow across the cross section of the Venturi tube and shift of the vapor clouds away from the walls can be suitable from the cavitation erosion point of view.

While the experimental data acquired during the investigation provide rich source of information, the additional CFD analysis was convenient due to the uninvestigated velocity field downstream the swirl generator information. Moreover, the numerical analysis of this phenomenon brings better insight into the dynamics of the cavitating structures complementary to the high-speed video records and other experimental data.

The following chapters consist of the experimental measurement description and following numerical investigation. The main scope of the paper will be focused on the combination of the experimental and numerical results to provide complex description of the cavitation induced by the presence of the swirl within the Venturi tube. The results are discussed in the direction from the supercavitation to the initial stage of cavitation.

\section{Experimental measurement}

Experimental measurement was performed exploiting closed cavitation circuit equipped with the pressure tank, where the pressure was set using the pressurized air and vacuum pump (fig. 1). Discharge within the circuit was set using the pump controlled by frequency convertor. This setup allowed to investigate different cavitation regimes for the constant discharge. Discharges between 4 and $7 \mathrm{l} / \mathrm{s}$ have been investigated with and without upstream mounted swirl generator.

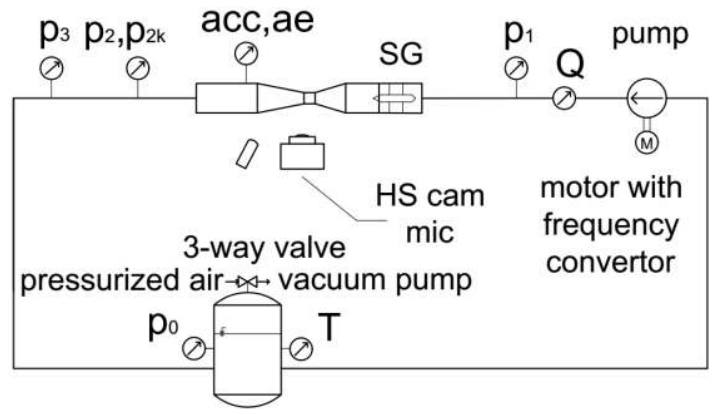

Figure 1 Scheme of cavitation circuit equipped with the transducers

The cavitation has been studied within the axisymmetric Venturi tube manufactured from plexiglass, thus it was possible to capture the investigated phenomenon using the high-speed (HS) camera. The main dimensions of the Venturi tube are depicted in the following figure 2 .

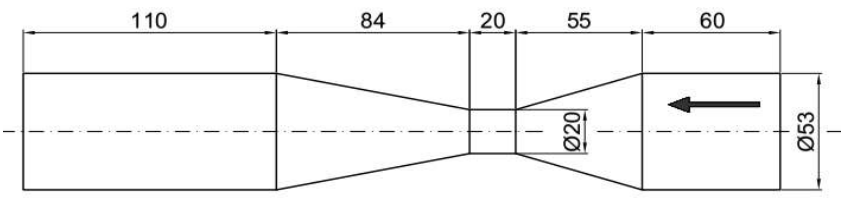

Figure 2 Main dimensions of the exploited Venturi Tube

Geometry of the swirl generator (SG) is depicted in the figure 3. Swirl generator consists of 9 fixed blades with the linear change of the trailing edge deviation angle from axial direction ( $30^{\circ}$ to $50^{\circ}$, from hub to shroud respectively).

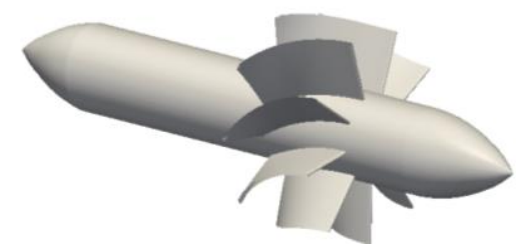

Figure 3 Model of the swirl generator

The various properties of the cavitating flow have been measured during the investigation as it is depicted in the Figure 1. While the data of acoustic emission and noise represent rich source of information, for the scope of this paper the pressure and vibration records as well as the high speed (HS) video records are important. These records, in conjunction with the record of the flow discharge $Q$ and water temperature $T$ enable to describe the static and dynamic properties of the cavitating flow. List of the important transducers is shown on the following table.

\begin{tabular}{|c|c|c|}
\hline Sgn. & Transducer & Type \\
\hline$p_{\mathrm{x}}$ & pressure & BD Sensors DMP 331 \\
\hline $\mathrm{p}_{2 \mathrm{k}}$ & pressure fluct. & Kistler - 211B4 \\
\hline $\mathrm{Q}$ & discharge & ELA - MQI 99 - SN \\
\hline $\mathrm{T}$ & water. temp. & Rawet - PTP 55 \\
\hline acc & vibrations & Aura SV129 \\
\hline
\end{tabular}

Table 1 List of the important transducers

The sampling frequency of Kistler and Aura transducer was $51.2 \mathrm{kHz}$, whereas the sampling frequency of the HS records was set to $20000 \mathrm{fps}$.

Grayscale H-S video records with the resolution of 1024 by 296 px have been captured using the Photron FastcamSA-X2 $1000 \mathrm{~K}$ camera. Venturi tube has was illuminated using two led matrix light sources using the dark background.

The amount of air within water has not been measured during the measurement. Test rig has been operated under the supercavitation regime prior the measurement for about 30 minutes. This method led to the final concentration between 6 to $8 \mathrm{mg} / \mathrm{l}$ of the oxygen based on our previous experience.

\subsection{Hydraulic loss coefficient evaluation}

Static characteristics of the flow have been evaluated using hydraulic loss coefficients. The additional properties such as water density $\rho$ and saturated vapor pressure $p_{v a}$ were calculated according to IAPWS based on the measured water temperature. It should be noted that the range of the investigated cavitation regimes was restricted by the test rig characteristics. The most problematic part of the rig represented pump shaft sealing, where the suction of air has been observed under certain circumstances.

To characterize the flow, the cavitation number $\sigma_{v e l}$ was evaluated (eq. 1). While the calculation of this value using bulk velocity within the throat of the nozzle $v_{t h}$ is reasonably accurate in case of the axial flow, in case of the SG the swirl significantly 
distorts velocity field. This issue will be in detail discussed in the part devoted to numerical simulations.

$$
\sigma_{\mathrm{vel}}=\frac{\mathrm{p}_{3}-\mathrm{p}_{\mathrm{vap}}}{0.5 \rho \mathrm{v}_{\mathrm{th}}{ }^{2}}
$$

Hydraulic loss coefficient $\xi$ has been evaluated using the formula (2).

$$
\xi=\frac{\mathrm{p}_{1}-\mathrm{p}_{3}}{0.5 \rho \mathrm{v}_{\mathrm{th}}^{2}}
$$

Using these results, it was possible to capture hydraulic loss coefficient evolution with respect to cavitation number. As it can be seen in the figure 4, the hydraulic loss coefficient is significantly higher in case of the SG presence. On the other hand, the significant amount of kinetic energy contained within the swirl is neglected. This discrepancy will be discussed in the chapter devoted to the numerical analysis. It should be also noted that the value of $\xi$ is not affected by the discharge considering individual investigated configurations.

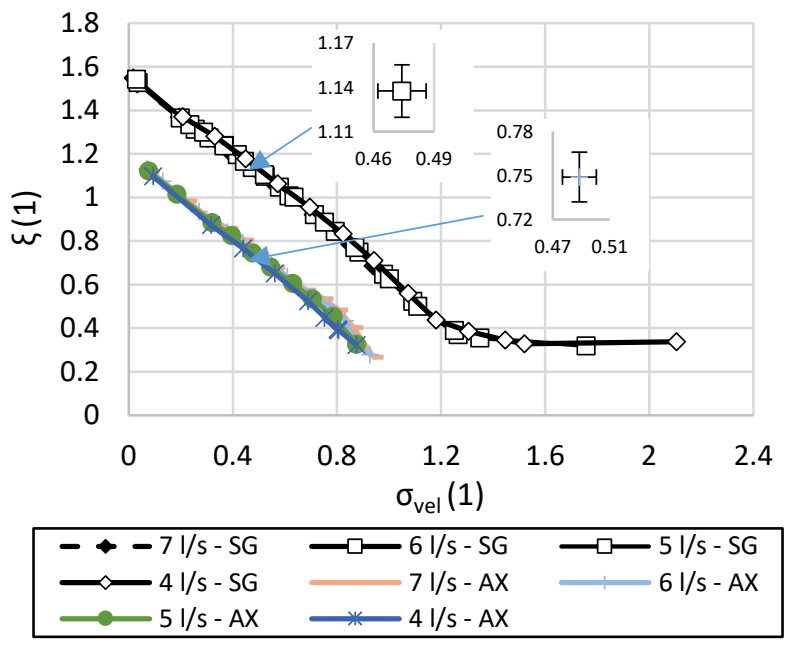

Figure 4 Hydraulic loss coefficient versus cavitation number (measurement uncertainty for selected operating points)

\subsection{Vibration induced by the cavitating flow}

The discussion of the cavitation dynamics will be focused mainly on the analysis of the test rig vibrations using the accelerometer and the analysis of $\mathrm{H}-\mathrm{S}$ records. It should be noted that the analysis will be done for the flowrates of $4 \mathrm{l} / \mathrm{s}$ and $6 \mathrm{l} / \mathrm{s}$. In general, it can be stated that the noise as well as vibrations of the rig were significantly higher in case of pure axial inflow (AX). While the influence of additional source of dampening presented by the SG cannot be fully neglected, the main reason for this behavior is significantly modified flow structure downstream the generator. These observations are supported by the analysis of the mean value of the pipe wall acceleration magnitude depicted in the figure 5 . Acceleration is imprint of the vibrations induced by collapsing vapor clouds.

The vibrations induced during the operation of the test rig under the supercavitation regime varied from 1.4 to $1.5 \mathrm{~g}$ while the maximum mean value of the vibrations reached $24.4 \mathrm{~g}$, corresponding to the axial inflow and $6 \mathrm{l} / \mathrm{s}$. It should be noted that the static value of measured acceleration on the test rig has was $0.14 \mathrm{~g}$, when the pump was turned off.

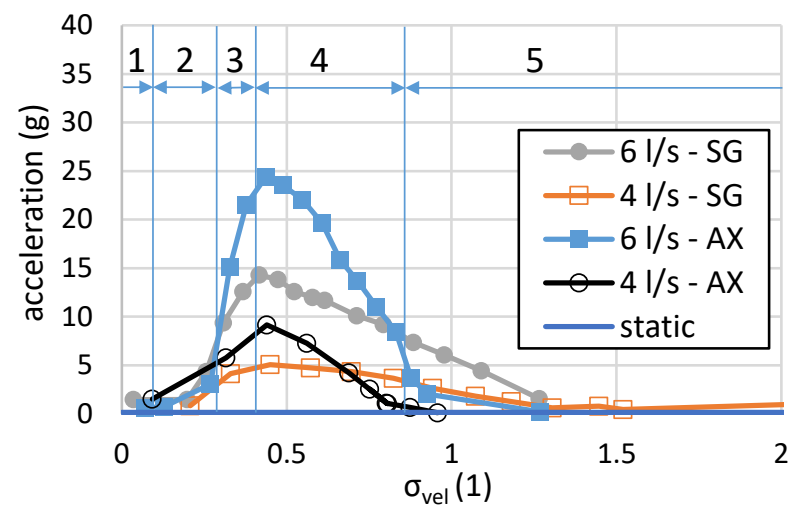

Figure 5 Analysis of test rig vibrations induced by cavitation

Increase of the acceleration magnitude related to the fully developed cavitation is steep in both of the investigated configurations (region 3).

The maximum magnitude of the acceleration has been significantly lower in case of the SG presence. The maximum vibrations have been observed between the fully developed and initial stage of cavitation. Above that these maximum values have been observed closed to the ovel value of 0.44 .

Seeing the decrease of the acceleration magnitude from the fully developed cavitation to the initial stage (region 4), it can be stated that the decrease is steeper in case of axial flow. Interesting observation is the fact, that the vibrations magnitude of the cavitating flow influenced by the additional swirl is higher compared to the axial flow down to a certain value of cavitation number. Unlike the value of cavitation number corresponding to the maximum acceleration, this threshold value is significantly less constant. In case of higher discharge $(6 \mathrm{l} / \mathrm{s})$, the vibrations of the axial flow became more significant close to the cavitation number of 0.83 , while in case of the lower discharge $(4 \mathrm{l} / \mathrm{s})$ this transition has been observed close to the cavitation number value 0.69 .

\subsection{High speed video records analysis}

Using the captured high-speed video records, it was possible to investigate typical cavitation patterns (Figure 6) for both test rig configurations. Beside the overall description of cavitation behavior, dominant frequencies of the cavitating structures were studied using the proper orthogonal decomposition (POD).

1.3.1. Cavitation regimes - influence of the induced swirl Region 1 in the Figure 5:

Supercavitation regime is typical for its low vibrations, pressure pulsations and noise induced by the cavitating flow regardless of the SG presence. Nevertheless, the cavitation patterns are completely different. In case of axial flow the liquid jet surrounded by the vapor was observed downstream the nozzle.

On the other hand, the coherent cavitating vortex surrounded by liquid water was observed in case of SG presence. Separation of the cavitating boundary layer occurred within the diffuser. 

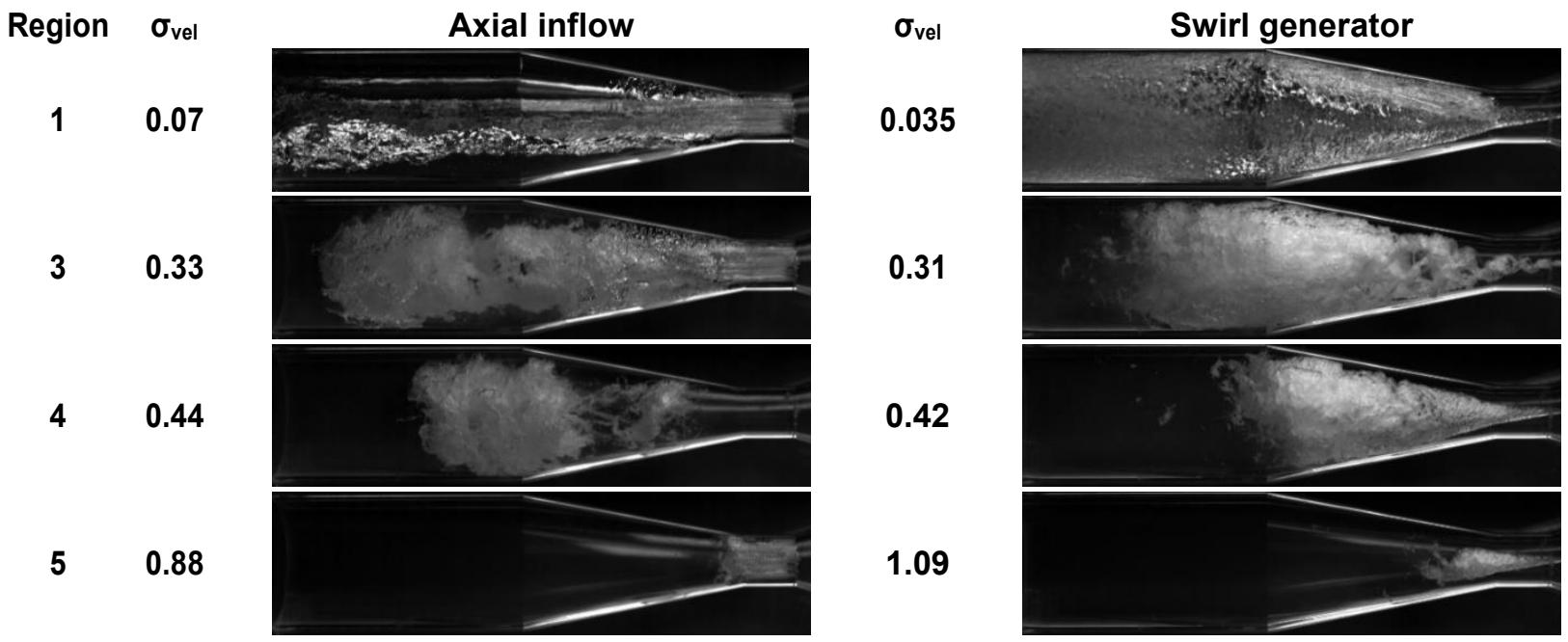

Figure 6 Set of images capturing cavitating structures and influence of the induced swirl over the wide range of $\sigma$.

Region 2 in the Figure 5:

Transition of the supercavitation to the fully developed regime of cavitation shows the alternation of the coherent liquid jet surrounded by the vapor phase and massive cavitating clouds in case of axial flow. Additionally, cavitating vortex induced by the SG is disturbed from time to time, while the backflow within the core of the structure can be observed prior this collapse. The observed magnitude of the mean pipe-wall acceleration remain low.

\section{Region 3 in the Figure 5:}

Fully developed cavitation is typical by periodical pulsations of the cavitating structures, which can reach significant length. The massive cavitating clouds were observed in case of the axial flow. The clouds are transported by the flow to the region of higher pressure, where clouds decay. Cavitating instabilities near the axis of the nozzle can be observed in case of the axial flow. Conical cavitating vortex was observed in case of SG presence. While its length remains relatively constant, the significant presence of small vortices can be seen in the cavitation closure region. Similar to the region 2, the backflow can be observed within the core of the vortex. When the backflow reaches throat of the nozzle the helical type of vortex breakdown occurs. Rapid decrease of the gaseous phase amount led to the decrease of fluid compliance and consequent rapid increase of pipe-wall acceleration up to the maximum value close to the region 4 .

Region 4 in the Figure 5:

Cavitation is characterized by periodical creation of the vortex rings in case of the axial flow. The lower is cavitation number, the more massive rings are. In case of the SG presence, the conical cavitating structure can be observed. The length of the structure is the shorter, the higher is the cavitation number. Stable nature of the cavitating structure affected by the swirl led to the more gradual decrease of the pipe-wall acceleration compared to the axial inflow.

Region 5 in the Figure 5:

In case of the axial flow, pulsations of the cavitating boundary layer in the throat of the nozzle have been observed as well as strongly stochastic instabilities of the closure region. With the additional swirl (SG), the straight cavitating vortex filament
(Fig. 7) has been observed downstream the generator. This filament became conically shaped with the decreasing cavitation number. Periodical helical type of vortex breakdown in throat of the nozzle was observed. Contrary to the previous regions, the pipe-wall acceleration is more significant in case of SG presence. In case of the axial inflow, the cavitating structures became relatively stable, especially in case of the highest investigated values of $\sigma_{v e l}$.

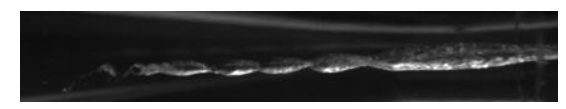

Figure 7 Detail of the straight cavitating vortex within the throat of the nozzle

\subsubsection{Proper Orthogonal decomposition}

Proper Orthogonal decomposition (POD) represents mathematical method of the dynamical phenomena investigation. Data are decomposed to the set of POD modes consisting of spatial coefficient $\varphi$ and temporal coefficient $a$. Beside the analysis of the experimentally obtained data, usually velocity field, it is possible to exploit this method for analysis of the video records. Since number of analyzed spatial points was rather high, so-called method of snapshots has been applied [8].

POD was utilized by Danlos et al. for study of passive control of cavitation using the surface grooves. Decomposition has been exploited for the detection of the cavitation regimes [9]. Another application of the POD can be found in investigation of aerated cavitation dynamics within the Venturi nozzle with rectangular cross-section [10]. In-depth investigation of the swirl structures downstream the runner of swirl generator designed by team at UPT led by Resiga was done by Štefan et al. [11]. Investigation of the swirling flow dynamics downstream the runner of Francis turbine using the POD was done by Rudolf et al. [12].

The results presented in this contribution are successive to the published analyses of the transitions between fully developed and supercavitation regime of cavitation Venturi tube [7]. POD analysis has been carried out considering the constrained region of interest containing the upper half of the 
captured images. The main reason for the image was to minimize influence of uneven scene illumination. For the purpose of the analysis, each $10^{\text {th }}$ image of record was considered. Most significant spatial coefficients of the pixels intensities fluctuations with and without the upstream SG corresponding to the initial, fully developed and supercavitation regimes are depicted in the figure 8 . Additional swirl significantly influences the spatial coefficients structure. This is especially noticeable in case of supercavitation, where the most important fluctuations are small instabilities close to interface layer and in case of initial phase of cavitation where the small but coherent axisymmetric vortex was observed in case of SG presence.

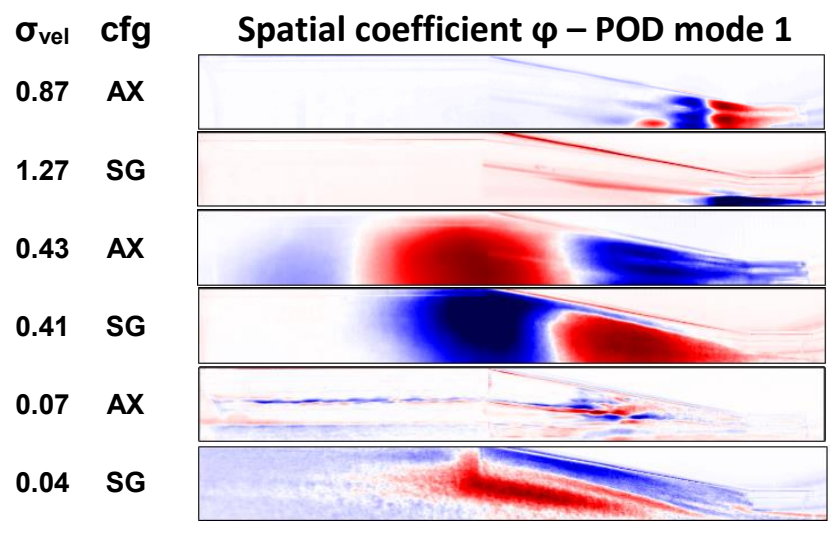

Figure 8 Spatial coefficients of the most significant POD modes red max values, blue - min values

Spatial coefficient and time coefficient define the variation of the POD mode significance in time. Thus, it is possible to study dynamics of the POD modes using the spectral analysis of the temporal coefficients. Dominant frequencies of the most significant POD modes versus $\sigma_{v e l}$ are depicted in the figure 9 . The frequencies are the higher, the higher is the discharge. Presence of the swirl induced by the SG increases the frequencies slightly. This increase of frequency is more significant in case of $6 \mathrm{l} / \mathrm{s}$. Comparing the results of the frequency analysis with the chart of the pipe wall acceleration magnitude, several observations can be described. In the region of fully developed and initial stage of cavitation, the growth of the frequency value is nearly linear. in case of transitions regimes different results can be found.

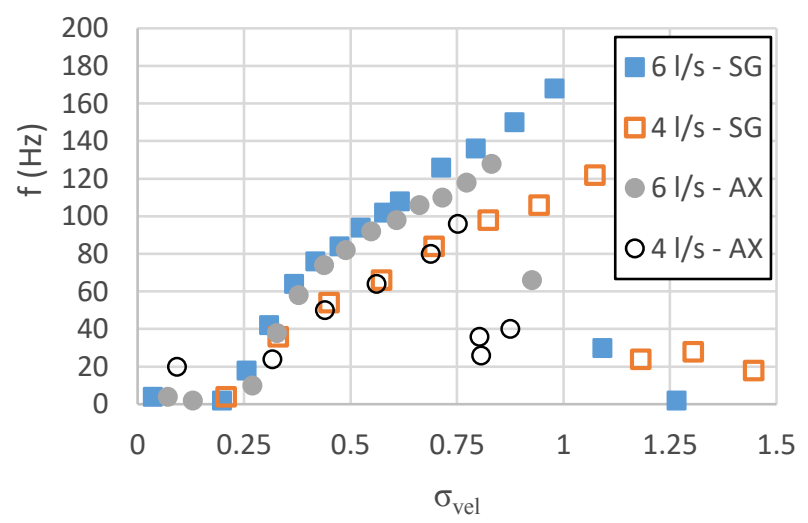

Figure 9 Dominant frequencies obtained by analysis of the temporal coefficients corresponding to the most important dynamic POD modes
Main source of instability represents highly stochastic structures of the interphase region in case of supercavitation. In the fully developed stage of cavitating flow, the increase of dominant frequencies is more rapid, compared to the initial stage of cavitation. Cavitation number range of this rapid frequency increase is in good agreement with the steep increase of the acceleration magnitude. Faster increase of the frequency magnitude, compared to the initial stage of cavitation can be attributed to the position of the frequency downstream the nozzle, or to the faster increase of the vapor phase amount within the flow and corresponding faster increase of the medium compliance. Both of these assumptions led to the conclusion that the decrease of the vapor phase volume is more rapid in case of the fully developed cavitation compared to the further decrease of the vapor amount during the initial stage of cavitation.

Increasing value of the frequencies is related to the decreasing mean magnitude of vibrations in the initial regime of cavitation. In other words, slower pulsation of massive cavitating structure produces stronger vibrations compared to the faster pulsations in this region. On the other hand, the lower is the cavitation number in case of fully developed cavitation, the lower is the observed magnitude of the acceleration.

With the increasing cavitation number, the abrupt drop in dominant frequency can be seen. Regardless of the frequency magnitude drop occurred, acceleration of vibrations remains still higher by order compared to the vibrations induced by supercavitation. It should be noted that these frequency drops occurred earlier in case of axial inflow.

Relative significance of the first dynamic modes computed as a fraction of the POD mode eigenvalue to sum of all eigenvalues is depicted in the figure 10 . Comparing with the chart of the acceleration magnitude, remarkable match of the results can be found. Cavitation number corresponding to the maximum relative significance is in good agreement with maximum of the mean acceleration magnitude. Above that, while in the wide range of operating regimes, the relative significance of the most important POD mode is higher in case of the axial flow. Decrease below the value obtained in case of SG presence can be found. On the other hand, it should be noted that $\sigma_{v e l}$ of this relative significance drop is higher, than in case of pipe wall acceleration analysis for $4 \mathrm{l} / \mathrm{s}$ discharge.

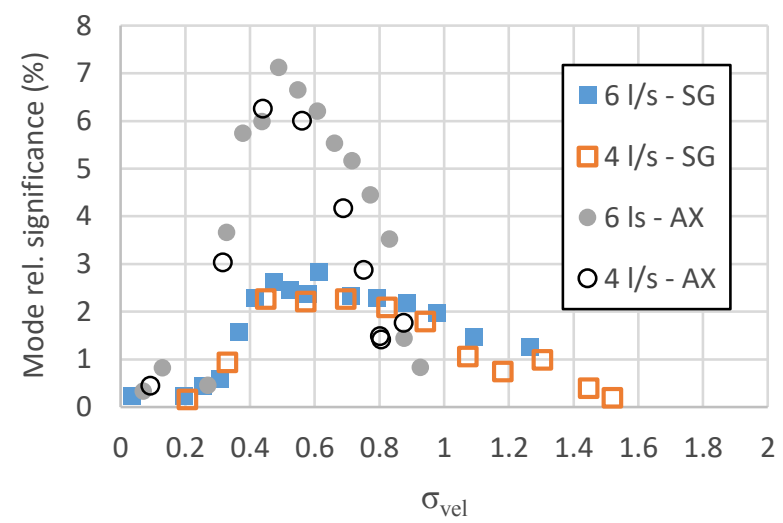

Figure 10 Relative significance of the most important dynamic POD modes contributions 


\section{Numerical analysis}

Numerical investigation has been carried out using OpenFoam 1606+ and its multiphase interPhaseChange solver based on VOF method. The main reason for the numerical investigation was to complement the experimental data, especially the velocity field downstream the generator. Nevertheless, the CFD results can provide much more. It should be emphasized that the numerical investigation will be focused on the configuration including SG. The numerical analysis of the cavitating flow within the investigated Venturi tube considering only axial inflow can be found in [6].

\subsection{Computational grids}

The computational grids were created using the cfMesh and its predominantly hexahedral algorithm cartesianMesh. The cfMesh provides wide range of the mesh refinement tools as well as boundary layers tools, while the process of the mesh properties setting is not complicated and mesh generation is relatively fast.

In the first step of the numerical analysis, the whole experimental section including the SG has been considered (figure 13). Two different computational grids were utilized for the numerical analysis: coarse (CG1) and refined (CG2). The comparison of the coarse and refined meshes is depicted in the figure 11.

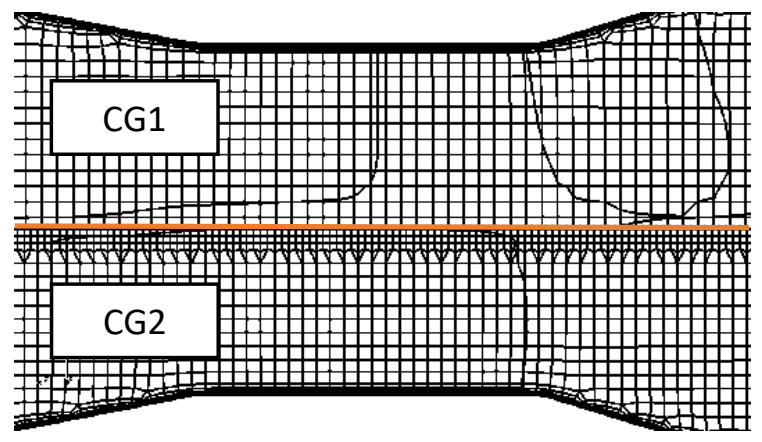

Figure 11 Comparison of the coarse (CG1) with the refined (CG2) computational grid - throat of the nozzle

Comparison of the computational grids size, as well as number of the hexahedral cells within the grids is depicted in the following figure 12 .

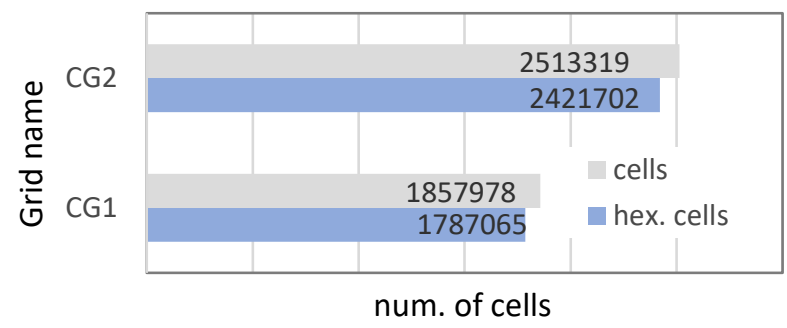

Figure 12 Computational grids

\subsection{Numerical settings}

The interPhaseChangeFoam solver was exploited. This solver is using so-called VOF method to capture interface of two incompressible, isothermal, immiscible fluids where the phase-change is considered. VOF method was proposed by Hirth. [13]

Beside the single continuity and momentum equations of the mixture, the transport equation of the volume fraction $\alpha$ had to be solved (eq. 3).

$$
\frac{\partial\left(\alpha \rho_{1}\right)}{\partial \mathrm{t}}+\nabla \cdot\left(\alpha \rho_{\mathrm{l}} \mathbf{U}\right)+\nabla \cdot\left[\alpha \mathbf{U}_{\mathrm{C}}(1-\alpha)\right]=\mathrm{R}_{\mathrm{e}}-\mathrm{R}_{\mathrm{c}}
$$

Where $R_{e}$ and $R_{c}$ represent the source terms of evaporation and condensation respectively. These terms are calculated using cavitation models, usually based on Rayleigh-Plesset equation.

Model proposed by Kunz et al was considered for the purpose of this investigation [14]. The choice was based on the reasonably accurate results of the previous numerical investigation considering axial inflow into the Venturi tube [7].

In case of Kunz cavitation model, the source terms $R_{e}$ and $R_{c}$ are computed using the formulas (4) and (5).

$$
\begin{gathered}
\mathrm{R}_{\mathrm{e}}=\mathrm{C}_{\mathrm{v}} \frac{\rho_{\mathrm{v}} \alpha}{\mathrm{t}_{\infty}\left(0.5 \rho_{\mathrm{l}} \mathrm{U}_{\infty}{ }^{2}\right)} \min \left[0, \mathrm{p}-\mathrm{p}_{\mathrm{v}}\right] \\
\mathrm{R}_{\mathrm{c}}=\mathrm{C}_{\mathrm{c}} \frac{\rho_{\mathrm{v}} \alpha^{2}(1-\alpha)}{\mathrm{t}_{\infty}}
\end{gathered}
$$

Where $t_{\infty}$ is mean flow time scale and $U_{\infty}$ represents mean stream velocity [14]. Values of the empirical constants $C_{v}$ $(900)$ and $C_{c}(12000)$ were set-up based on [6]. The realizable $k-\varepsilon$ model has been chosen for the numerical analysis. The model of turbulence was derived from the standard $k-\varepsilon$ model by Shih et al. [15]. The proposed modifications of turbulent viscosity formulation and transport equation for the dissipation rate led to the significantly lower turbulence dampening. This model was successfully applied by Štefan et al for the swirl dissipation investigation within the diffuser downstream the SG [3]. While this research involved only the single-phase computations, the good results were obtained by main author in case of the cavitating flow in Venturi tube (considering axial inflow) [6]. Numerical simulations of the multiphase flow within the Venturi tube for the purpose of discharge measurement using Star-ccm+ and realizable k- $\varepsilon$ was done by Brinkhorst [16].

Inlet velocity has been set according to the experimentally measured discharges. The inlet turbulent properties were prescribed using the turbulentMixingLengthDisssipationRatelnlet in case of $\varepsilon$ and turbulentIntensityKineticEnergylnlet with $5 \%$ of intensity in case of $k$.

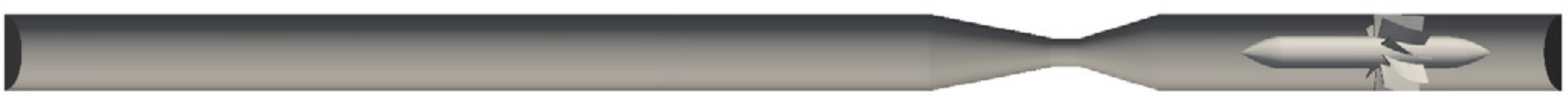

Figure 13 Computational domain 
Near wall regions have been treated by kqRWallFunction in case of $k$ and epsilonWallFunciton in case of $\varepsilon$. Velocity noSlip boundary condition has been set on the walls.

The outlet pressure has been set according to the static pressure experimentally measured by transducer $\mathrm{p} 3$ placed $700 \mathrm{~mm}$ downstream the nozzle.

\subsection{Evaluation of the hydraulic losses}

As it has been mentioned, the bulk velocity within the throat of the nozzle, which was considered as a reference velocity for the computation of $\sigma_{v e l}$ as well as for $\xi$, neglects the tangential and radial components of the velocity. These components are significantly higher in case of the swirl induced by the SG. Loss coefficient analysis based on the bulk velocity is depicted in the figure 14 .

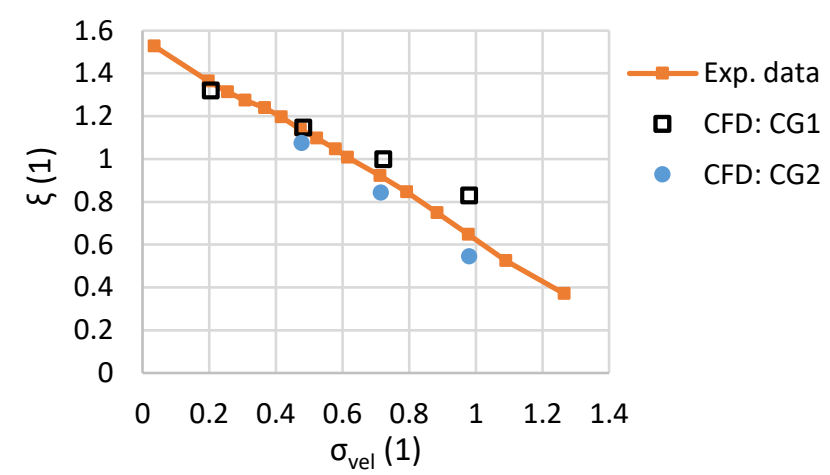

Figure 14 Hydraulic loss coefficient - influence of the computational grid

It is clearly depicted that the results of the CFD analysis are significantly closer to the experimental data in case of refined grid CG2. While the decrease of the hydraulic loss coefficient is linear considering both of the utilized computational grids, the inclination is closer to the experimental data in case of the CG2. For these reasons, the CG2 has been picked as reference for the further investigation.

The energy correction coefficient $\alpha_{\text {corr }}$ given by equation (6) has been evaluated in the inlet of the nozzle throat. This region was chosen as a reference due to the stability of the flow.

$$
\alpha_{\text {corr }}=\frac{\iint \mathrm{v}_{\mathrm{ax}} \cdot \mathrm{v}_{\mathrm{mag}}^{2} \mathrm{dS}}{\mathrm{v}_{\text {bulk }}{ }^{3} \mathrm{~S}}
$$

Where $v_{a x}$ represents axial velocity, $v_{\text {mag }}$ is velocity magnitude and $v_{\text {bulk }}$ is bulk velocity.

The evaluated mean value of $\alpha_{\text {corr }}$ is 1.173 based on the analysis of three operating points utilizing the CG2. The values of $\alpha_{\text {corr }}$ were nearly constant and the difference between the lowest and highest value was less than $0.5 \%$. Only slight increase of $\alpha_{\text {corr }}$ was observed for increasing $\sigma$. The $\alpha_{\text {corr }}$ evaluated in case of the axial inflow is 1.01.

The analysis of the hydraulic loss coefficients as well as $\sigma_{v e l}$ have been corrected using the $\alpha_{c o r r}$ in case of $6 \mathrm{l} / \mathrm{s}$ and the $S G$ presence to respect real kinetic energy magnitude. The results are depicted in the following figure 15.

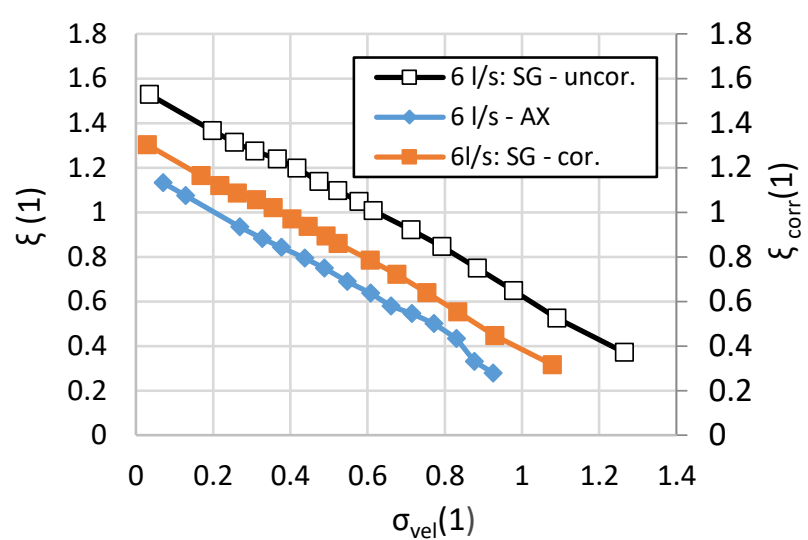

Figure 15 Correction of the hydraulic loss coefficient using the energy correction factor

It is clearly depicted that the values of the corrected hydraulic loss coefficients are approximately lower by 0.22 compared to the uncorrected results. Due to the fact that this decrease is devoted to the inclusion of the swirl within the throat, it is plausible to assume that the remaining difference between the hydraulic loss curves of the corrected results and the results of the axial inflow analysis is caused mainly by the presence of the swirl generator.

If the corrected values of the cavitation number are applied for the pipe wall acceleration magnitude analysis, the discrepancy between the peaks positions of maximum vibrations for the axial flow and flow affected by the induced swirl can be discerned (figure 16).

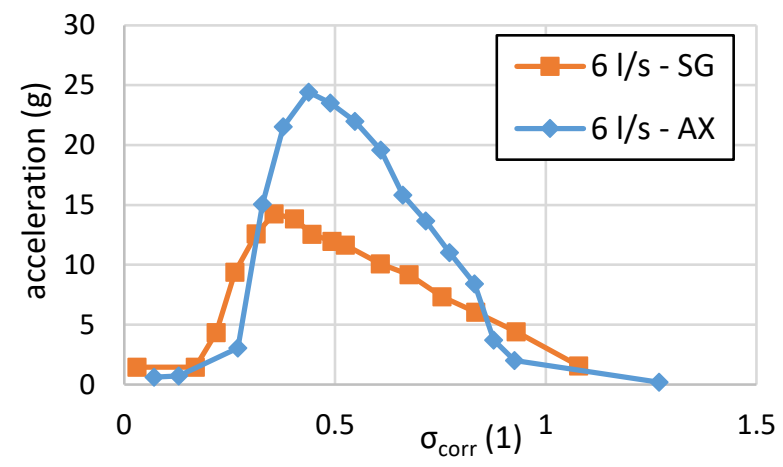

Figure 16 Pipe wall acceleration magnitude versus cavitation number corrected using $\alpha_{\text {corr }}$

As it can be seen in the figure 16, the cavitation number corresponding to the maximum acceleration magnitude is lower by $33.5 \%$ in case of SG presence compared to the axial inflow.

To discuss this result, another definition of the cavitation number based on the pressure difference was exploited (eq. 7). This definition of cavitation number is also widely used in case of cavitation analysis within valves. The results are depicted in the figure 17.

$$
\sigma_{\text {pres }}=\frac{p_{3}-p_{\text {vap }}}{p_{1}-p_{3}}
$$




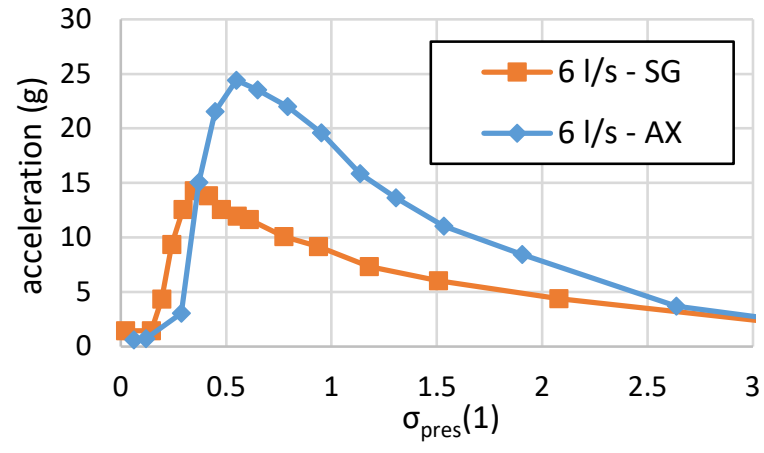

Figure 17 Pipe wall acceleration magnitude versus $\sigma_{\text {pres }}$

Several conclusions can be drawn comparing the figures 16 and 17. Similar to the correction of $\sigma_{\text {vel, }}$, the cavitation number $\sigma_{\text {pres }}$ corresponding to the maximum magnitude of acceleration is lower in case the SG presence. On the other hand, the observed difference is $48.2 \%$ between the investigated experimental configurations in case of $\sigma_{\text {pres }}$, which is certainly larger difference compared to the correction by $a_{c o r r}$. Regardless this difference, the results obtained using the corrected $\sigma_{v e l}$ are in good qualitative agreement with the analysis of the acceleration exploiting $\sigma_{\text {pres. }}$.

\subsection{Discussion of the numerical results}

The value of the swirl number $S n$ evaluated according to the eq. 8 at the inlet of the throat downstream the SG is 0.234 .

$$
\mathrm{Sn}=\frac{\int \mathrm{v}_{\mathrm{ax}} \cdot \mathrm{v}_{\mathrm{tan}} \mathrm{rdS}}{\mathrm{R} \int \mathrm{v}_{\mathrm{ax}}^{2} \mathrm{dS}}
$$

Where $v_{\tan }$ represents tangential velocity, $r$ radial coordinate and $R$ is a reference radius (i.e. radius of the throat).

Although the results of the numerical analysis were in reasonably good agreement with the experimental observations from the hydraulic loss point of view, two major differences of the flow fields should be described. Thin cavitating vortex filament was observed downstream the spike of SG during the experiments (Figure 18). Using the abovementioned settings and computational grids, the vortex filament has not been captured numerically. On the other hand, the significant pressure drop in the region of the nozzle axis can be seen in the figure 19 and above that the contour of the averaged vapour void fraction $(0.25)$ extends into the diffuser upstream the throat of the nozzle. The second qualitative discrepancy between the numerical and experimental flow downstream the swirl generator is represented by the periodical pulsations of the cavitating boundary layer within the diffuser downstream the throat of the nozzle observed in case of the CFD analysis (Figure 19). The cavitation in this region was relatively seldom in case of the experiments. The significant cavitation of boundary layer was observed only during the supercavitation regime in experimental observation.

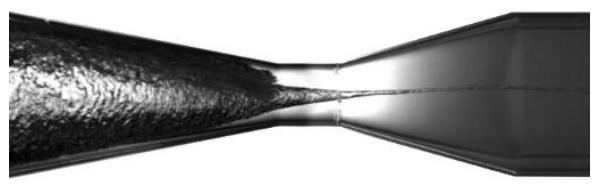

Figure 18 Cavitating vortex filament downstream the SG

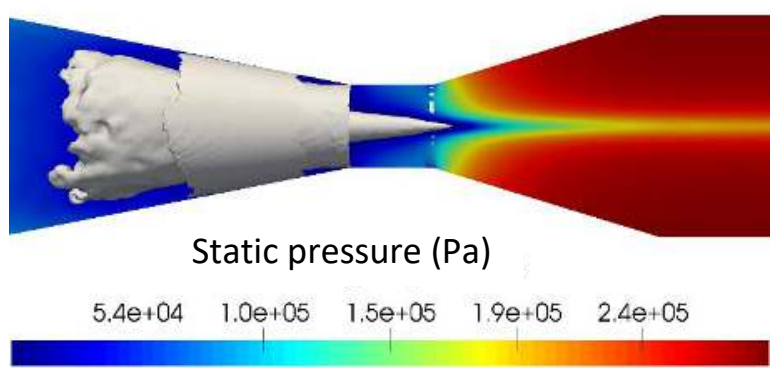

Figure 19 Flaws of the numerical simulation: absence of the cavitating vortex filament and significant cavitation of the boundary layer downstream the throat of the nozzle.

The absence of the cavitating vortex filament in case of CFD could be partially devoted to the rough surface of the real swirl generator, where the small imperfections of the spike play an important role in the process of the cavitation nucleation. On the other hand, the overestimated cavitation of the boundary layer points to slightly different redistribution of the flow downstream the nozzle throat in case of CFD analysis. Regardless the abovementioned flaws of numerical computations, the obtained results are in good agreement with the experimental observations.

Downstream the coherent conical cavitating structure, the relatively short, but significant, helical vortex can be found in case of $\mathrm{H}-\mathrm{S}$ image corresponding to the cavitation number of 0.98 (fig. 20). It was possible to capture this phenomenon using the refined computational grid CG2 which is depicted in the figure 11 using the vapour void fraction of 0.25 .

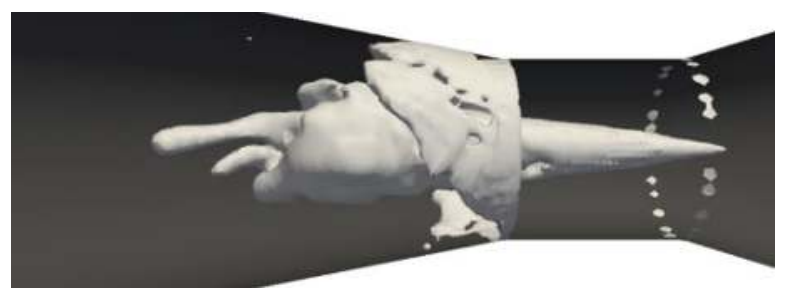

Figure 20 Cavitating vortex captured using the CFD

The helical vortex breakdown in the throat of the nozzle observed during the experimental measurement was also captured using the CFD (figure 21). Again, the contour of 0.25 of vapour void fraction was exploited for the cavitation visualization.

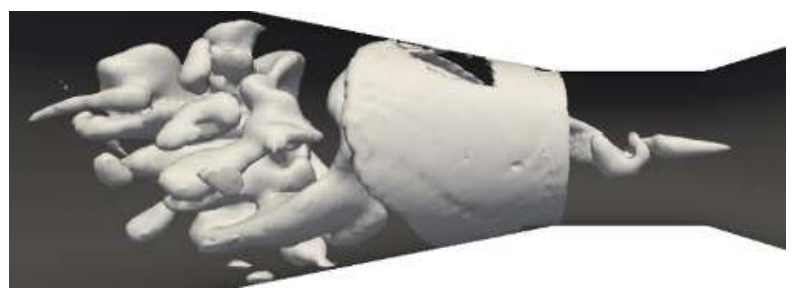

Figure 21 Helical vortex breakdown captured using the CFD

The comparison of the axial velocity and vapour void fraction fields with the images captured using the HS camera is provided in the following set of images (figure 22). The images correspond to the maximum length of the cavitation structures, prior the beginning of its collapse. 
$\boldsymbol{\sigma}_{\mathrm{vel}}$

HS image

(HS/CFD)

$0.98 / 0.98$

$0.71 / 0.71$

$0.47 / 0.48$
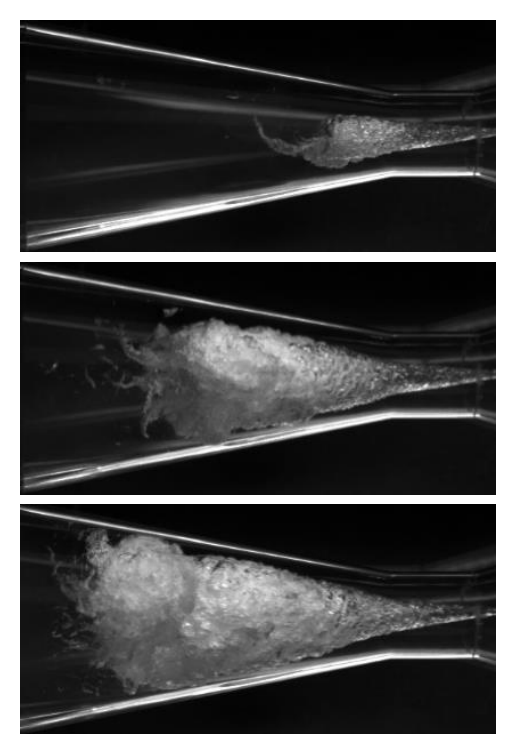

Vapour void fraction (1)
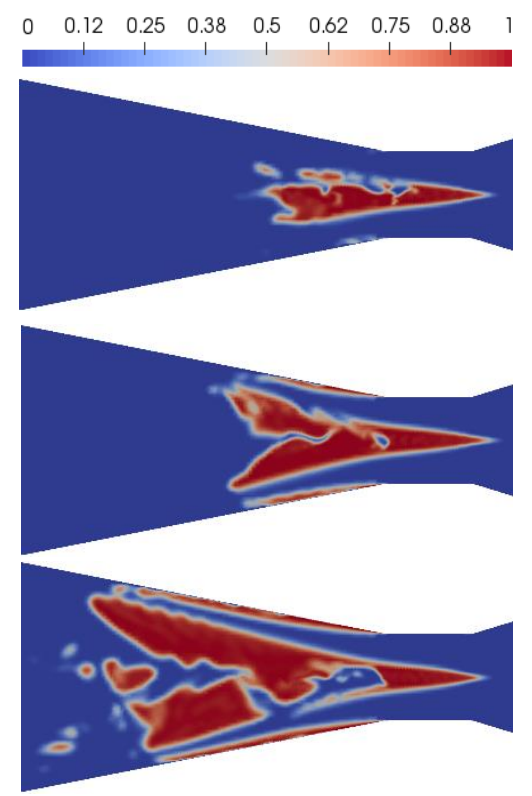

Axial velocity $\left(\mathrm{ms}^{-1}\right)$

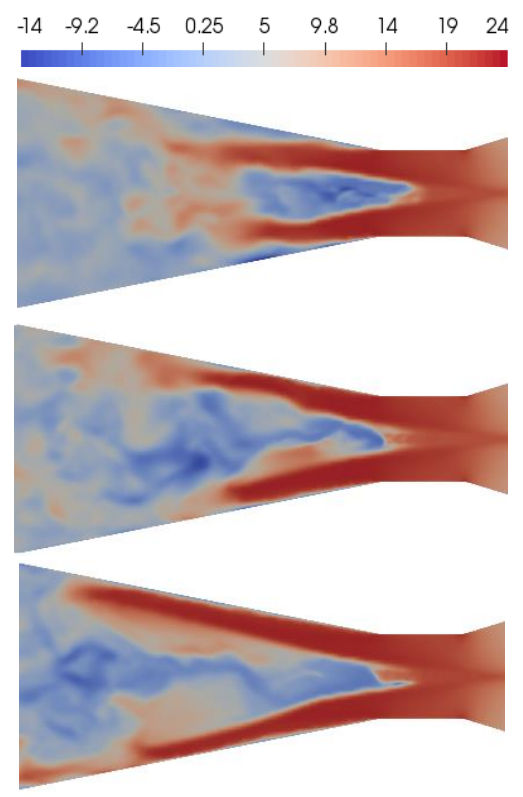

Figure 22 The images captured using HS camera in comparison with the numerical results.

First of all, it should be emphasized that while the HS images represent 3D instantaneous spatial distribution of the cavitation within the flow, the images of $2 \mathrm{D}$ longitudinal crosssection was chosen for the numerical results visualization. As it is clearly depicted, the amount of the gaseous phase is the higher, the lower is cavitation number. Above that, the predicted length of the cavitating structures prior the beginning of their collapse is in good agreement with the experimental observation.

Several conclusions can be stated, comparing the water volumetric fraction and field of axial velocity. The region of the significant backflow can be found within the core of the cavitating vortex, which has been also observed on the HS records.

As it is clearly depicted in the set of figures 22 , the significant amount of liquid water can be found within the core of cavitating vortex in case of the cavitation numbers of 0.71 and 0.48 . While the $\mathrm{H}-\mathrm{S}$ records do not provide information of the phase distribution across the cross-section of the nozzle, it was possible to observe that the liquid water was sucked in to the cavitating vortex in the cavitation closure region (Figure 23).

$$
\text { Axial velocity }\left(\mathrm{ms}^{-1}\right)
$$

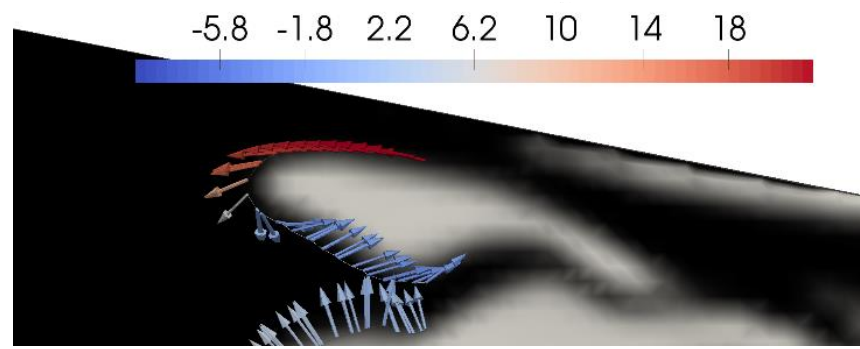

Figure 23 Detail of the cavitation closure region with velocity vectors colored by the axial velocity magnitude $\left(\mathrm{ms}^{-1}\right)$.

\section{CONCLUSIONS}

The cavitation within the Venturi tube has been investigated using the experimental and numerical methods with particular regard to effect of the induced swirl on the cavitating flow properties.

The magnitude of the average pipe wall acceleration has been significantly higher in case of the axial inflow. For both of the investigated configurations it was possible to observe steep increase of the pipe wall acceleration magnitude during the fully developed cavitation. In the certain instant, the acceleration magnitude reached the maximum value and then started to decrease for both investigated configurations. The hydraulic loss coefficients have been also analyzed. It was clearly depicted that while the inclination of the hydraulic loss coefficients curve versus cavitation number was nearly the same for both of the configurations, the values were significantly higher in case of the swirl generator presence. These results were subsequently corrected using the numerical methods.

The dynamics of the cavitating structures was investigated exploiting the POD. The dominant frequencies of the most significant dynamic modes were found. As it was clearly depicted, the increase of the dominant frequencies was steeper during the fully developed cavitation compared to the initial stage. Thus, it is possible to assume that the decrease of the cavitation volumetric, as an additional source of compliance within the flow, is faster in this stage.

Relative significance of the most important dynamic modes has been studied using the analysis of the corresponding eigenvalues. Results were in surprisingly good agreement with the results of the acceleration magnitude analysis. Consequently, the considerable mutual interdependence of the coherent cavitation fluctuations with the magnitude of pipe wall acceleration (i.e. vibrations) can be assumed. 
The numerical analysis carried out using the OpenFoam $1606+$ and its multiphase interPhaseChangeFoam solver provided rich source of additional information. The influence of the computational grid has been discussed using the analysis of the hydraulic loss coefficient, where good agreement with the experimental data was obtained using the finer mesh. Therefore, the finer mesh has been chosen for the further analysis. Using the evaluation of the kinetic energy correction factor at the inlet part of the throat of the nozzle, the experimental results were corrected in case of the swirl generator presence. This correction has been done due to the fact, that the bulk velocity (and consequently kinetic energy) exploited for the $\xi$ and $\sigma_{\text {vel }}$ computation is significantly lower compared to the actual velocity magnitude in case of the flow affected by the presence of the swirl.

Using the corrected data, the hydraulic losses as well as acceleration magnitude were analyzed. In case of pipe wall acceleration magnitude, the difference between $\sigma_{v e l}$ corresponding to the maximum vibrations based on the test rig configuration was found. This result was confirmed using alternative definition of the cavitation number, where similar discrepancy of the acceleration peaks has been observed. Thus, it is possible to assume, that this approach is correct.

From the qualitative point of view, two major flaws of the CFD simulations were described, absence of the cavitating filament downstream the spike of swirl generator and overestimated cavitation of the boundary layer downstream the throat. Other properties such as length of the cavitating structures and presence of the specific structures were captured correctly. The redistribution of the flow downstream the throat has been described using the CFD.

Overall, it can be stated that the cavitating flow within the Venturi tube is significantly modified when the swirl is introduced. The cavitating patterns are completely different compared to the axial inflow. Dominant frequencies of the axial pulsations induced by cavitation are slightly higher in case of SG presence, while the vibrations are significantly more severe in case of axial inflow. Cavitation of the boundary layer was considerably less significant and the volumetric of the cavitation was surrounded by the liquid water in case of SG presence. Thus, it can be assumed, that the surfaces of nozzle are far less threatened by the occurrence if the cavitation erosion compared to the axial inflow.

\section{ACKNOWLEDGMENTS}

Czech Science Foundation is gratefully acknowledged for support of this research under project No 16-18316S „Principles and mechanisms causing microorganism elimination by hydrodynamic cavitation ".

\section{REFERENCES}

[1] Ch. E. Brennen. Cavitation and Bubble Dynamics. Oxford University Press, 1995.

[2] P. Dörfler, M. Sick M., A. Coutu. Flow-induced pulsation and vibration in hydroelectric machinery: engineer's guidebook for planning, design and troubleshooting. Springer, 2013.

[3] D. Štefan, P. Zubík, M. Hudec, P. Rudolf. Numerical and experimental investigation of swirling flow in a conical diffuser. EPJ Web of Conferences, 92, 2015

[4] D. Jančura, P. Mikula, B. Maršálek, P. Rudolf, F. Pochylý. Selective method for cyanobacterial bloom removal: hydraulic jet cavitation experience. Aquaculture International, 22:509-521, 2014.

[5] P. Rudolf, M. Hudec, M. Gríger, D. Štefan. Characterization of the cavitating flow in convergingdiverging nozzle based on experimental investigation. EPJ Web of conferences, 67, 2014.

[6] J. Kozák, P. Rudolf, D. Štefan, M. Hudec, M. Gríger. Analysis of Pressure Pulsations Of Cavitating Flow In Converging-Diverging Nozzle. 6th IAHR International Meeting of the Workgroup on Cavitation and Dynamic Problems in Hydraulic Machinery and Systems, 2015.

[7] J. Kozák, P. Rudolf, D. Štefan, R. Huzlík, M. Hudec et al. Transition of cavitating flow to supercavitation within Venturi nozzle - hysteresis investigation, EPJ Web of Conferences, 2017.

[8] G. Berkooz, P. Holmes, J. Lumley, The proper orthogonal decomposition in the analysis of turbulent flows. Annual Rev. of Fluid Mechanics 25:539-575, 1993.

[9] A. Danlos, F. Ravelet, O. Coutier-Delgosha, F. Bakir, C. Sarraf, F. Bakir. Cavitation regime detection through Proper Orthogonal Decomposition:Dynamics analysis of the sheet cavity on a grooved convergentdivergent nozzle, International Journal of Heat and Fluid Flow,47, 2014.

${ }^{[10]}$ P. Tomov, A. Danlos, S. Khelladi, F. Ravelet, C. Sarraf, F. Bakir. POD study of aerated cavitation in a venturi nozzle. Journal of Physics: Conference Series, 656, 2015.

[11] D. Štefan, P. Rudolf, S. Muntean, R. Susan-Resiga. Proper Orthogonal Decomposition of Self-Induced Instabilities in Decelerated Swirling Flows and Their Mitigation Through Axial Water Injection. ASME. J. Fluids, 139(8), 2017.

[12] P. Rudolf, D. Štefan. Decomposition of the swirling flow field downstream of Francis turbine runner. IOP Conference Series: Earth and Environmental Science, ISSN: 1755-1315, 2012.

[13] C.W. Hirt, B.D. Nichols. Volume of fluid (VOF) Method for the dynamics of free boundaries. J. of computational physics, 39:201-225, 1981.

[14] R.F.Kunz, D.A. Boger, D.R. Stinebring, T.S. Chyczewski, J.W. Lindau, H.J. Gibeling, S. Venkateswaran, T.R. Govindan. A Preconditioned Implicit Method for Two-Phase Flows with Application to Cavitation Prediction. Computers and Fluids, 2000.

[15] T.-H. Shih, W.w. Liou, A. Shabbir, Z. Yang, J. Zhu. A New K-epsilon Eddy Viscosity Model for High Reynolds Number Turbulent Flows: Model Development and Validation. NASA Technical Memorandum, 106721, 1994.

[16] S. Brinkhorst, E. Lavante, G. Wendt. Experimental and Numerical Investigation of the Cavitation-induced Choked Flow in a Herschel Venturi-Tube. Flow Measurement and Instrumentation 54, 2016. 